\title{
Study the Effect of Different Parameters and Improving the Shielding Effectiveness of a Metallic Enclosure with Extra Wall
}

\section{Sedigheh Vaezikakhki, Seyed Saeid Mosavinejad, Mehdi Bahadorzadeh}

Faculty of Engineering, Islamic Azad University, Mashhad, Iran

\section{Email address:}

s.vaezi@mshdiau.ac.ir (S. Vaezikakhki), mosavinejad@mshdiau.ac.ir(S. S. Mosavinejad), m.bahadorzadeh@mshdiau.ac.ir(M.Bahadorzadeh)

\section{To cite this article:}

Sedigheh Vaezikakhki, Seyed Saeid Mosavinejad, Mehdi Bahadorzadeh. Study the Effect of Different Parameters and Improving the Shielding Effectiveness of a Metallic Enclosure with Extra Wall. American Journal of Electrical and Computer Engineering. Vol. 3, No. 2, 2019, pp. 53-57. doi: 10.11648/j.ajece.20190302.11

Received: April 21, 2019; Accepted: July 23, 2019; Published: August 14, 2019

\begin{abstract}
This paper propose a new method for improving shielding effectiveness (SE) of a rectangular enclosure with multiple circular apertures. In this method in order to compensate the effects of the apertures on (SE) parameter, we use two metallic walls each containing apertures instead of the one wall. By using two walls for shielding an enclosure, the shielding effectiveness of the enclosure will improve. The numerical simulation uses a symmetric condensed node of TLM - TD (Transmission line Modeling Method - Time Domain) and subsequent Fourier Transform and the result was verified by HFSS and CST software. We obtain the shielding effectiveness response to an electric field impulsive excitation. It is shown that field polarization and enclosure direction has a noticeable effect on SE that should be considered in SE calculations. The effect of different parameters and improving the shielding effectiveness of a metallic enclosure with extra wall is studied. An optimization of the influence of the place of apertures in the walls and the diameter of circles on two walls is presented.
\end{abstract}

Keywords: Shielding Effectiveness, EMC, TLM, Extra Shielding Wall

\section{Introduction}

Nowadays, regarding the electromagnetic immunity and electromagnetic interferences of the electronic devices increase the importance of a careful design of shielding enclosures.

There are different routes for coupling unwanted signals into shielding structures. Apertures, Seams and slots are common path for coupling the energy. Since usually there is a sort of coupling energy into shielding boxes we need to define the quality of a shielding box by introducing the shielding effectiveness (SE) of the enclosure.

EMC regulations demands the specific level of shielding effectiveness for shielding structures to meet the defined standards. The shielding effectiveness is defined as the ratio of received power without shielding to the received power with shielding.

$$
S E=20 \log \left(\frac{E_{S}}{E_{0}}\right)
$$

A number of computational methods have been proposed for the solution of shielding problems, including the finite-difference time-domain (FDTD) method [1], the method of moments (MOM) [2], and the finite element method (FEM) [3]. The Shielding effectiveness metallic enclosure with a square aperture of dimension $60 \times 60 \mathrm{~mm}$ is analyzed by Transmission Line Method (TLM) in [4] and is improved with using two metallic walls in [5].

\section{Shielding Effectiveness Analysis}

Figure 1 shows a typical metallic enclosure with extra shield screen and a circular aperture of diameter $\mathrm{w}$ illuminated by a plane wave. All sides are assumed perfectly conducting with an infinitesimal thickness. The observation point is located at a distance $\mathrm{p}$ away from the aperture inside the enclosure and on a line normal to the aperture and passing through the center of the enclosure. The internal dimensions of enclosures are assumed $120 \mathrm{~mm} \times 300 \mathrm{~mm} \times 300 \mathrm{~m}$. 


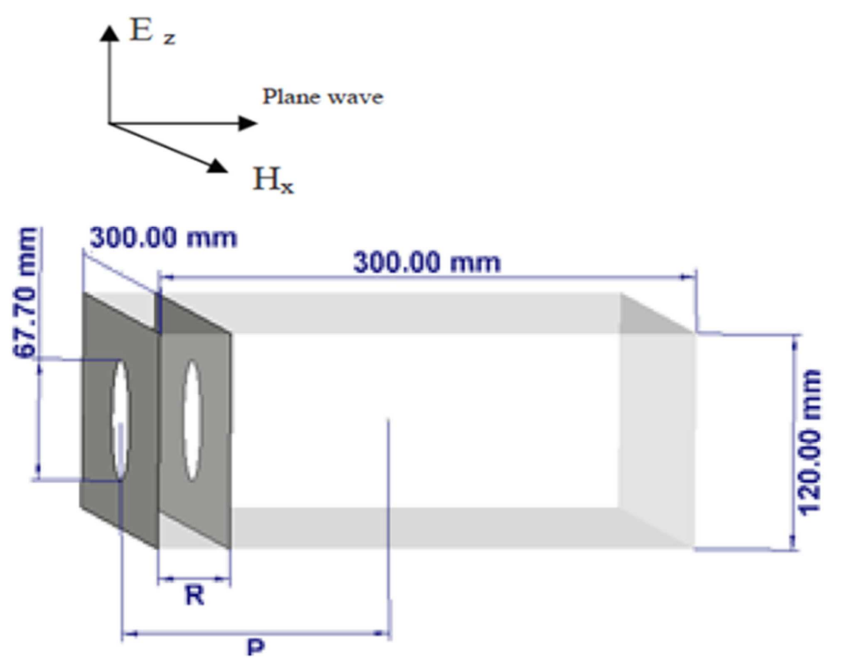

Figure 1. A typical enclosure with an extra shield screen.

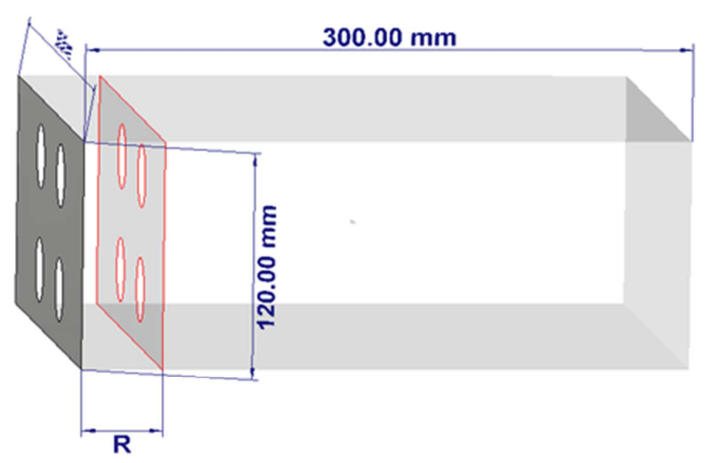

(a)

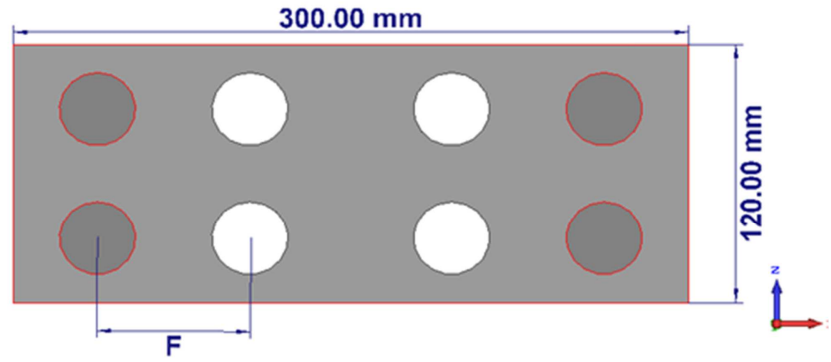

(b)

Figure 2. (a) Front panel of enclosure and the place of apertures on two walls. (b) Displacement of apertures.

For all simulations, in order to produce plane wave which is radiated to enclosure a free space volume of $120 \mathrm{~mm} x$ $300 \mathrm{~mm} \times 100 \mathrm{~mm}$ is placed in front of the aperture. A simple absorbing boundary condition is applied in the free space region. The results for a single screen and proposed structure is shown in Figure 2. This results show that using second wall improves shielding effectiveness of the enclosure, but resonant frequency of the enclosure increases because according to the equation for a closed rectangular metallic cavity (2) the dimensions of enclosure has been reduced.

$$
f_{r}=\frac{c}{2} \sqrt{\left(\frac{m}{a}\right)^{2}+\left(\frac{n}{b}\right)^{2}+\left(\frac{p}{d}\right)^{2}}
$$

As it has been shown in [5-7] using multiple apertures will improve shielding effectiveness of enclosure. In this structure, also using multiple apertures will increase SE parameter. To investigate this matter we used four circular apertures instead of main aperture that each of them has surface $(\mathrm{r}=16.93 \mathrm{~mm})$ almost equal to a quarter of the main aperture $(\mathrm{r}=67.70 \mathrm{~mm})$ surface Figure 2. The distance between two walls in all simulations were kept constant $\mathrm{R}=20 \mathrm{~mm}$.

\section{Results}

A. Effect of aperture, wall and displacement.

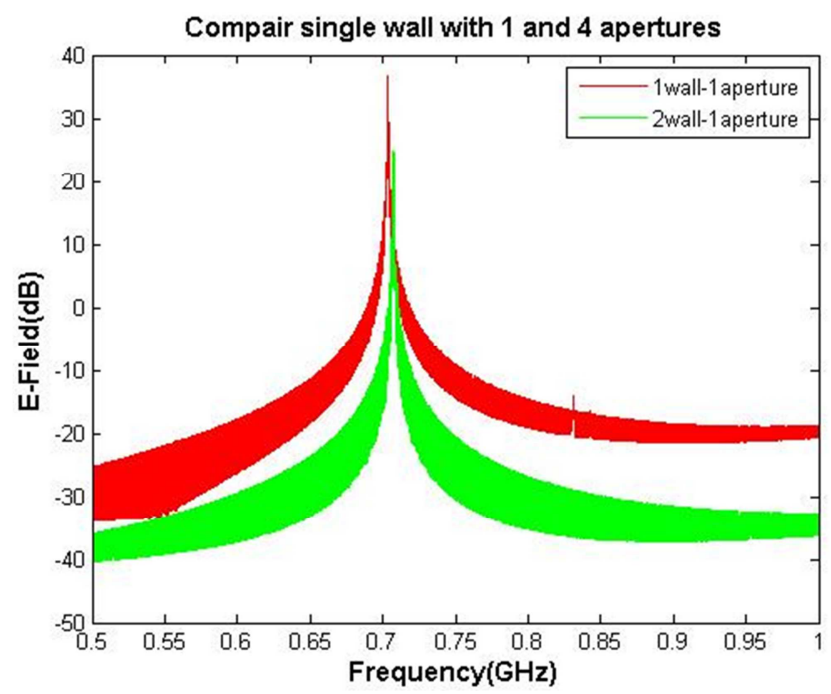

Figure 3. Effect of internal wall and apertures on SE.

As seen in the Figure 3, it is quite evident with the conversion of the entrance apertures into four apertures and then the use of the second wall the shielding effectiveness will improve. The effect of the displacement of apertures in second wall was also investigated and it showed that displacement makes shielding effectiveness better. Results for three different displacement is presented in Figure 4.

By using four aperture the level of E-field inside the enclosure reduces about $12 \mathrm{~dB}$ and will increase SE parameter and displacement makes $\mathrm{SE}$ about $24 \mathrm{~dB}$ better for $\mathrm{F}=40$.

$B$. Effect of radiation field polarization

In the real case field enters the enclosure from all direction. Therefore, the polarization, incident and azimuth angles should be considered. Effect of radiation field polarization on shielding effectiveness of enclosure with two shielding walls and four circular apertures studied. As shown in Figure 5 a shielding box with mentioned dimensions was simulated.

By keeping the enclosure constant $(\varphi=0)$ and changing the polarization of radiation, observe that the SE depends on the radiation wave polarization, and has decreased as polarization changes from the vertical to the horizontal. In all cases, the resonance frequency remains constant but for some polarization, we have second resonance frequency about 900MHz. 


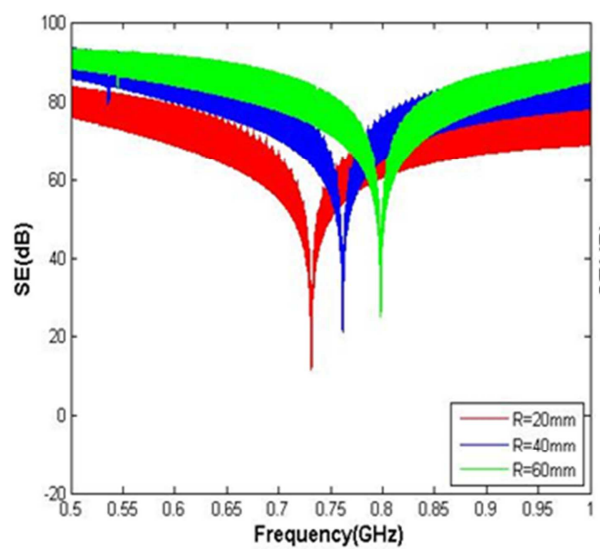

(a)

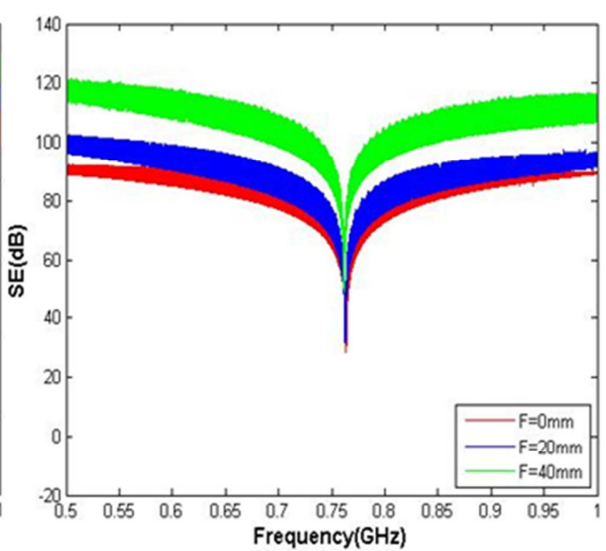

(b)

Figure 4. (a) Shielding effectiveness of the enclosure for the three different distances between walls. (b) Shielding effectiveness of the enclosure for three different displacement.
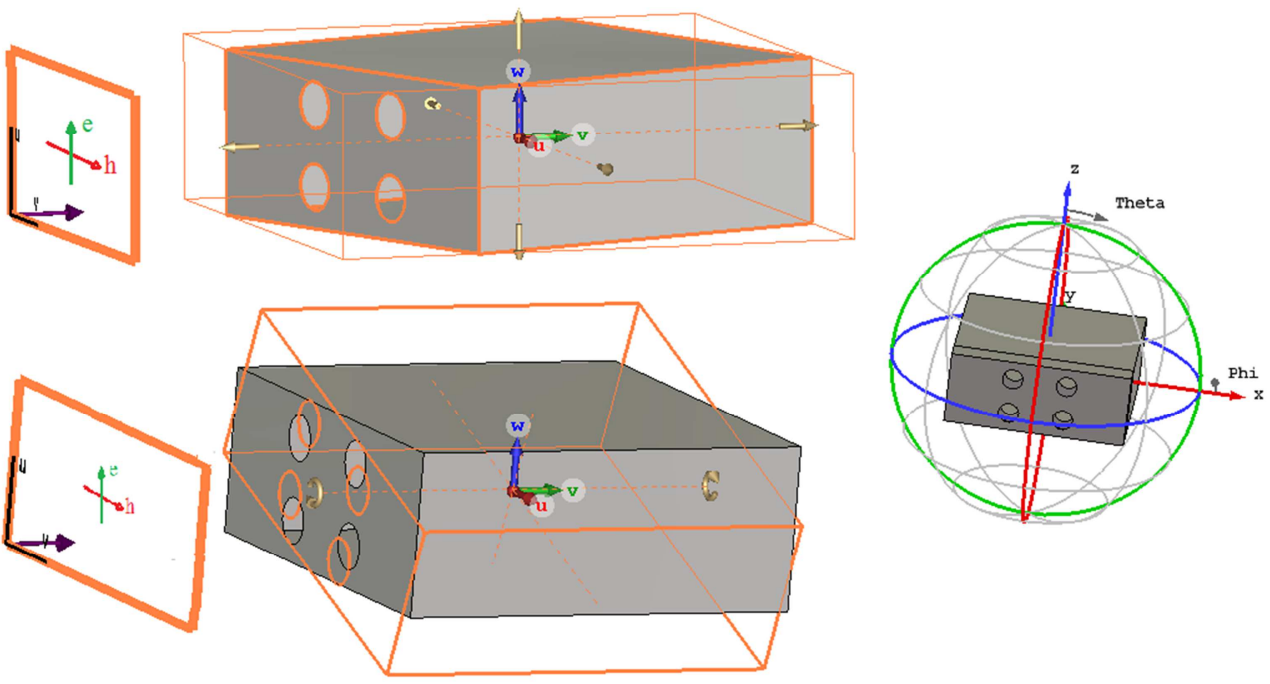

Figure 5. Geometry of rectangular enclosure with apertures and direction of electric field.

C. Effect of Oblique Incidence or direction of enclosure

By keeping the polarization constant $(\theta=0)$ and changing the direction of enclosure, as shown in Figure 6, we can also see the variation of $\mathrm{SE}$ as the angle of the incident wave changes; as it becomes larger, the value of SE increases. We observe that the SE depends on the angle between radiation

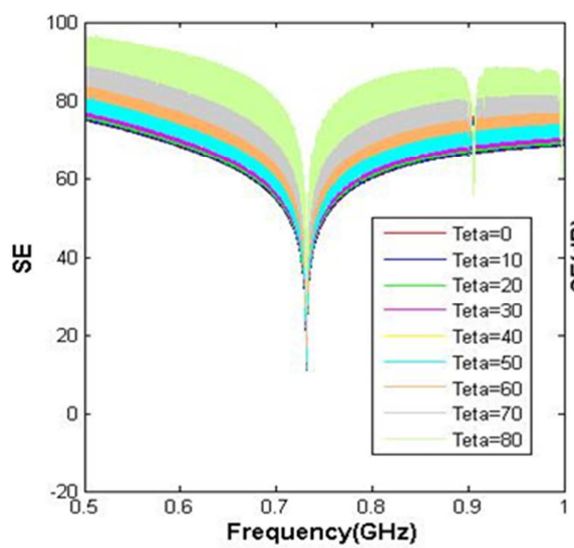

(a) Variable $(\Theta)$ wave and enclosure, and has increased as angle of field with wall with four apertures changes from zero to ninety. In all cases, the resonance frequency remains constant.

D. Optimization of distance bitween apertures by genetic algorithm.

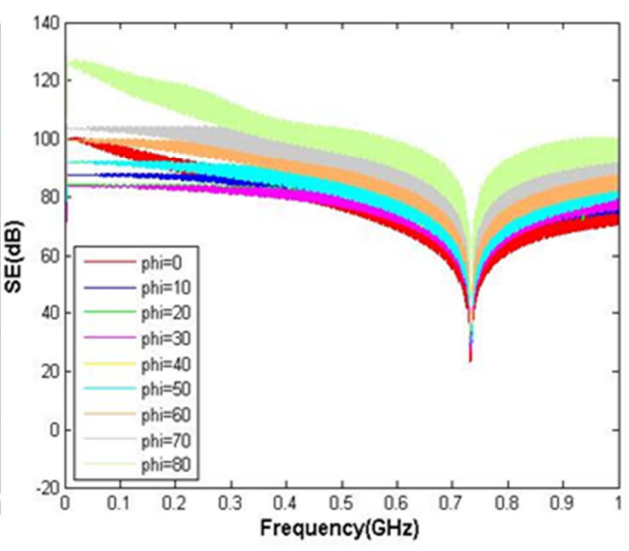

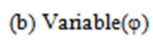

Figure 6. Effect of angle between field and enclosure on SE for double wall shielded enclosure for $R=40 \mathrm{~mm}$. 
In order to obtain the lowest field inside the enclosure and the highest amount of shielding effectiveness, we are using GA optimization method to find the optimum distance between the apertures as shown in Figure 7, the optimum distance is $\mathrm{F}=262.492 \mathrm{~mm}$.

Furthermore, CST software was used to find the Furthermore, CST software was used to find the optimum size for distance between apertures and the results represent a good agreement. As shown in Figure 8 shielding box with mentioned distance between apertures was simulated. Changing the displacement in vertical direction or z-axis does not have any effect on SE and resonance frequency.

\section{Conclusions}

In this paper, an enclosure with circular apertures in front wall is simulated based on transmission line matrix method. The effect of changing the various parameters on the shielding effectiveness has been investigated. It is shown that field polarization and enclosure direction has a noticeable effect on SE that should be considered in SE calculations. It has also been noted that SE parameter can be improved by displacement of apertures, changing the distance between the two walls and between apertures. The optimum distance between apertures by genetic algorithm has been achieved.

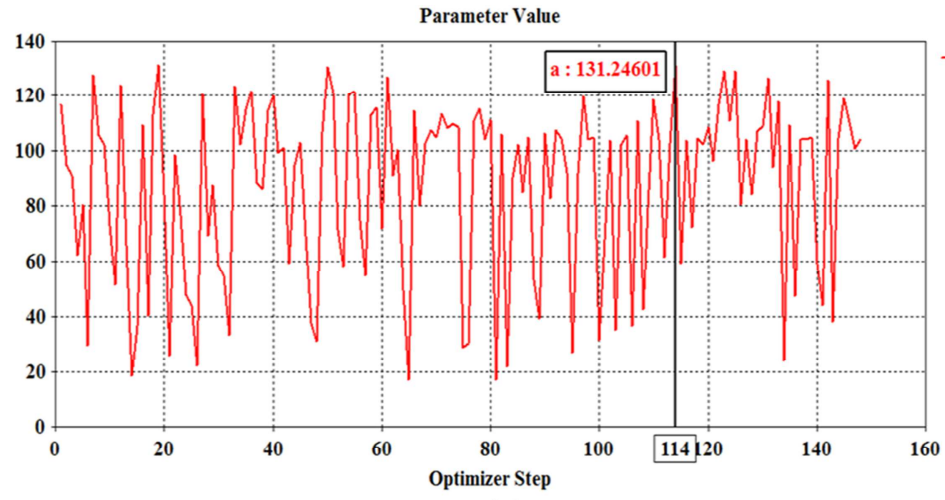

(a)

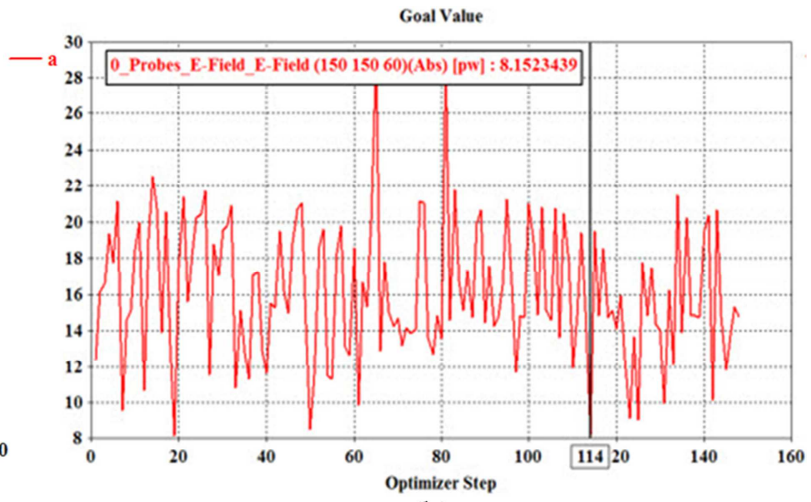

(b)

Figure 7. Genetic algorithm optimization by parameter of the distance between the apertures.

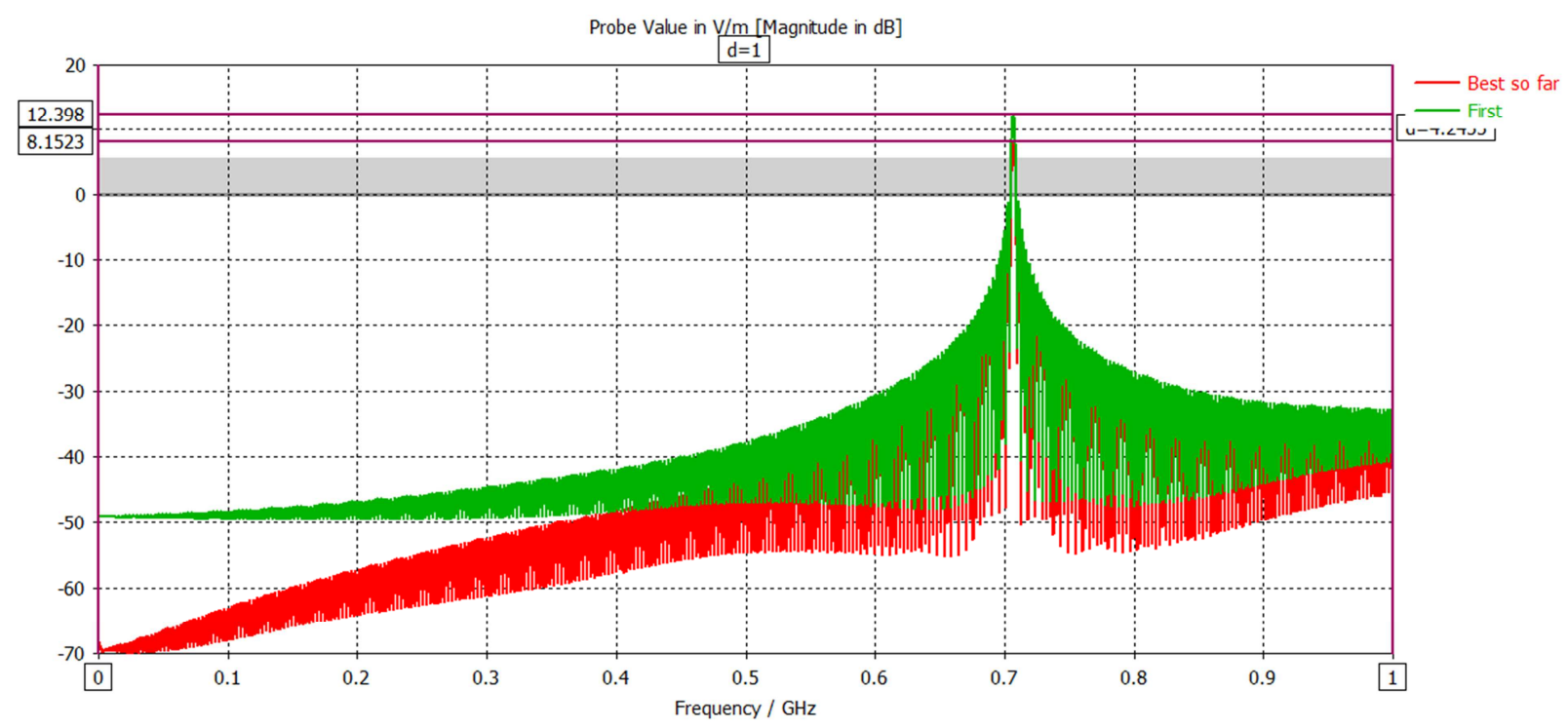

Figure 8. Field inside the enclosure for main and optimum distance between apertures.

\section{References}

[1] G. Cerri, R. D. Leo, V. M. Primiani, and M. Righetti, "Field penetration into metallic enclosures through slots excited by ESD," IEEE Trans. Electromagnetic Compatibility, Vol. EMC-36, No. 2, pp. 110-1 16, May. 1999.

[2] G. Cerri, R. D. Leo and V. M. Primiani, "Theoretical and experimental evaluation of the electromagnetic radiation from apertures in shielded enclosures," IEEE Trans
Electromagnetic Compatibility., Vol. EMC-34, No. 4, pp. 423-432, November 1992.

[3] M. Edrisi and W. K. Chan, "EMC methodology for numerical electric field computation inside enclosure with aperture," Electronics Letters, Vol. 35, pp. 1233- 1235, 1999.

[4] A. R Attari, K. Barkeshli, "Application of the Transmission Line Matrix Method to the Calculation of the Shielding Effectiveness for Metallic Enclosures," Palais des congrès Acropolis, France, November12-14, 2002. 
[5] M. Bahadorzadeh, M. Naser. Moghaddasi, "Improving the shielding effectiveness of a rectangular metallic enclosure with aperture by using extra shielding wall," IEEE Trans. Montreal, QC, July 2006.

[6] J. L. SilveiraP, S. Benhassine', L. Pichon' and A. Raizer, ” Analysis of the shielding effectiveness of a rectangular enclosure with apertures by TLM-TD," Fourth International Conference on Computation in Electromagnetics (CEM 2002), 8-11 APRIL 2002, BOURNEMOUTH, UK.

[7] M. Bahadorzadeh, A. A. Lotfi-Neyestanak "A novel and efficient technique for improving shielding effectiveness of a rectangular enclosure using optimized aperture load Authors" Journal Elektronika ir Elektrotechnika, Volume 18, Issue 10, Pages 89-92.

[8] A. Shourvarzi, and M. Joodaki, "A network of ports to estimate shielding effectiveness of an enclosure with apertures," in Proc. Electromagn. Compat. Eur., 2016, pp. 626-630.

[9] A. Shourvarzi, and M. Joodaki, "Using Aperture Impedance for Shielding Effectiveness Estimation of a Metallic Enclosure with Multiple Apertures on Different Walls Considering Higher Order Modes," IEEE Trans. Electromagnetic Compatibility, vol. PP, issue: 99, 2017.

[10] A. Shourvarzi, and M. Joodaki, "Shielding effectiveness estimation of an enclosure with an arbitrary shape aperture", EMC Europe 2017.

[11] Nie, B. L. and P. A. Du, "An efficient and reliable circuit model for the shielding effectiveness prediction of an enclosure with an aperture," IEEE Transactions on Electromagnetic Compatibility, Vol. 57, No. 3, 357-364, 2015.

[12] Yin, M. C. and P. A. Du, "An improved circuit model for the prediction of the shielding effectiveness and resonances of an enclosure with apertures," IEEE Transactions on Electromagnetic Compatibility, Vol. 58, No. 2, 448-456, 2016.

[13] Dehkhoda, P., A. Tavakoli, and M. Azadifar, "Shielding effectiveness of an enclosure with finite wall thickness and perforated opposing walls at oblique incidence and arbitrary polarization by GMMoM," IEEE Transactions on Electromagnetic Compatibility, Vol. 54, No. 4, 792-805, 2012.

[14] S. Celozzi, R. Araneo, G. Lovat, Electromagnetic shielding, Hoboken, New Jersey: Wiley \& Sons, pp. 42-46, 2008.

[15] M. P. Robinson, T. M. Benson, C. Christopoulos, J. F. Dawson, M. D. Ganley, A. C. Marvin, S. J. Porter, D. W. P. Thomas, "Analytical formulation for the shielding effectiveness of enclosures with apertures", IEEE Trans. Electromagn. Compat., vol. 40, no. 3, pp. 240-248, Aug. 1998.

\section{Biography}

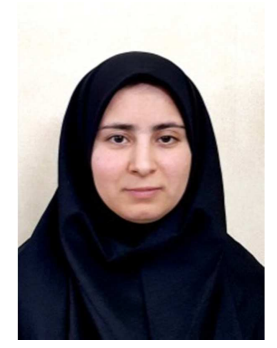

Sedigheh Vaezikakhki received her B. S. degree in electrical communication engineering in 2006, and the M. S. in telecommunication engineering - field theory from Khavarn University in Mashhad-IRAN in 2009. She is currently working toward the $\mathrm{Ph}$. D. degree at the Islamic Azad University in Mashhad-IRAN. Her main interests are microstrip antenna and Electromagnetic Compatibility and Shielding of circuits.

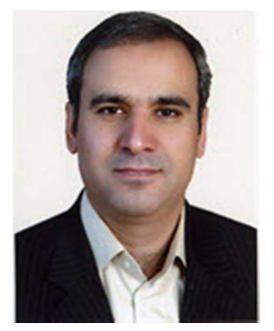

Seyed Saeid Mosavinejad received his B. S. degree in electrical communication engineering from Communication Faculty, Tehran, Iran, in 2000, and the M. S. in telecommunication engineering - field theory from Islamic Azad University in Tehran-IRAN in 2005. He started his cooperation with TCI as microwave and optic designer. $\mathrm{He}$ is currently working toward the $\mathrm{Ph}$. D. degree at the Islamic Azad University in Mashhad-IRAN. His main research area is design of antenna, Biosensor and Electromagnetic Compatibility and using new numerical method techniques for EMC problems.

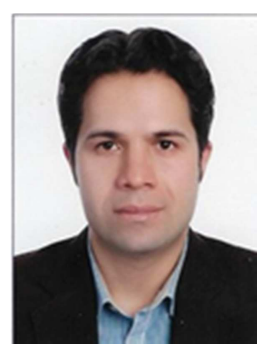

Mehdi Bahadorzadeh received his $\mathrm{PhD}$ in Telecommunication Engineering - Field theory in Science and Research Campus of Islamic Azad University in Tehran-IRAN in 2006 and he started his cooperation with IAUM as assistant professor in 2007. As a research scholar, he has been with George Washington university ECE department since 2015 to 2016 and currently he is cooperating with Oklahoma State University as a Post-Doctoral Fellow since 2017. Mr. Bahadorzadeh has researched in different area of applied electromagnetic and numerical methods. He is a contributor to many international journals with applied electromagnetic and antenna and microwave topics. 